\title{
Les pratiques de qualité dans les entreprises manufacturières et de services connexes : le cas des entreprises du Nouveau-Brunswick ${ }^{1}$
}

\author{
Yves Robichaud ${ }^{2}$, Université Laurentienne \\ Egbert McGraw, Université de Moncton \\ Mohamed Djerdjour, State University of New York
}

\section{Introduction}

Partout dans le monde, les entreprises réalisent de plus en plus que le concept de qualité est un élément essentiel pour leur survie dans le marché global d'aujourd'hui. Plus spécifiquement, les avantages d'une gestion efficace de la qualité sont nombreux : outre les bénéfices liés à l'amélioration de la qualité des produits et des services et à l'augmentation de la productivité, une gestion plus efficace de la qualité permet de diminuer le gaspillage et les coûts d'usinage, de réaliser une plus grande accessibilité au marché, de
La qualité a toujours été présente dans les entreprises, mais a surtout connu un développement rapide durant les vingt dernières années. qualité, on comprend facilement pourquoi la gestion de la qualité est devenue primordiale pour la survie des PME.

Tous ces éléments sont à la base de la nécessité d'approfondir nos connaissances sur la gestion de la qualité et des systèmes de qualité au sein des PME. C'est dans cette perspective que cet article examine l'état actuel des pratiques de qualité dans les petites et moyennes entreprises manufacturières et de services connexes du NouveauBrunswick. Par la même occasion, les données collectées à l'échelle de la capturer de meilleures affaires sur les marchés étrangers et d'améliorer les relations avec les employés, les clients et les fournisseurs. Bref, la gestion de la qualité permet à une entreprise d'être plus compétitive et d'améliorer sa performance organisationnelle ${ }^{3}$.

Or, les recherches sur la qualité ont tendance à se centrer surtout sur les entreprises de grande taille. Il existe, en effet, peu de littérature sur la gestion de la qualité et sur les systèmes de qualité au sein des petites et moyennes entreprises. Ce n'est que tout récemment que le concept de qualité dans les PME est devenu un sujet de recherche plus important ${ }^{4}$. Plusieurs facteurs peuvent expliquer ce phénomène. Mentionnons entre autres le nombre croissant des petites et moyennes entreprises, leur impact sur l'économie en général et le lien étroit qu'elles entretiennent avec les grandes entreprises. De plus, la globalisation des marchés engendre une compétition accrue qui impose de nouvelles normes aux PME œuvrant sur ces marchés. Si l'on ajoute à cela les exigences plus grandes des clients et des fournisseurs en matière de province du Nouveau-Brunswick contribueront à la rareté des données démographiques sur le sujet.

De façon générale, l'intérêt de cette recherche est de permettre aux responsables d'entreprises d'approfondir leurs connaissances sur les systèmes de qualité ainsi que sur les difficultés rencontrées lors de leur implantation. À ce sujet, nous espérons que les résultats de cette étude sensibiliseront les dirigeants d'entreprise aux avantages d'une gestion efficace de la qualité et sur la nécessité de l'application d'une telle démarche dans leur entreprise.

\section{Origine et évolution du concept de qualité}

La qualité a toujours été présente dans les entreprises, mais a surtout connu un développement rapide durant les vingt dernières années. Par exemple, au début du vingtième siècle, la gestion de la qualité consistait simplement à inspecter des produits afin d'assurer leur conformité aux spécifications. Puis, dans les années 1940, la qualité est devenue plus sophistiquée 
dans son application. On utilisait alors des techniques d'échantillonnage statistique pour évaluer le niveau de conformité des produits aux spécifications et des cartes de contrôle pour surveiller la performance des processus de production. Après la fin de la Deuxième Guerre mondiale, l'américain W. Edwards Deming, reconnu comme le père fondateur du contrôle de la qualité, conseillait plusieurs entreprises japonaises sur la façon d'améliorer la qualité de leurs produits. Cela permit au Japon de concevoir un modèle innovateur et unique de gestion de la qualité. Dans les années 1970, ce modèle avait tellement progressé qu'il existait une marge évidente entre la qualité des produits japonais et celle des produits nord-américains, les produits japonais étant d'une qualité nettement supérieure.

Ce n'est que plusieurs décennies plus tard que les entreprises américaines commencèrent à adopter la philosophie de Deming. Ainsi, vers le début des années 1980, les dirigeants des entreprises nord-américaines réalisèrent que le concept de qualité devait être considéré plus sérieusement et qu'ils avaient un problème avec leurs processus opérationnels. Cependant, le remède n'était pas facilement identifiable. D'abord, il existait à l'époque très peu d'informations sur la gestion de la qualité, et un sentiment patriotique exagéré empêchait certains responsables de chercher conseil auprès d'experts étrangers. De plus, la signification du terme " qualité » n'était pas très claire, ce qui prêtait facilement à confusion. Ce facteur, combiné à l'apparition de nombreuses méthodes de gestion de la qualité, compliquait davantage la situation. Enfin, le fait que l'adoption d'un système de qualité constituait un processus long et onéreux incitait peu les gestionnaires à s'aventurer dans une démarche de gestion de la qualité ${ }^{5}$.

Malgré ces difficultés, certaines entreprises nordaméricaines avaient déjà réussi, vers le milieu des années 1980, à implanter des systèmes de qualité, notamment le système de gestion intégrale de la qualité, l'équivalent en anglais du «Total Quality Management » (TQM). Depuis lors, plusieurs outils, techniques et méthodes ont été développés pour permettre aux organisations d'améliorer la qualité de leurs processus opérationnels et, conséquemment, la qualité de leurs produits et/ou services. Parmi ceux-ci, mentionnons le contrôle total de la qualité (CTQ), le contrôle statistique des processus et les normes ISO 9000. D'autres systèmes visant à contrôler de façon plus réactive la qualité ont aussi fait leur apparition : les cercles de qualité, l'échantillonnage statistique et les diverses méthodes d'inspection. C'est ainsi que le concept de qualité a continué de progresser pour devenir de nos jours un outil stratégique de valeur remarquable, voire indispensable.

Dans ce qui suit, nous présentons la méthodologie, puis nous ferons état des résultats obtenus. De façon plus précise, les résultats suivants sont présentés : les différences démographiques des PME manufacturières et de services connexes du Nouveau-Brunswick possédant un système de qualité et celles ne possédant pas de système de qualité, les raisons ou les motifs qui ont incité les entreprises à se munir ou non d'un système de qualité, les impacts positifs découlant de la mise en place d'un système de qualité et les difficultés rencontrées durant l'implantation.

\section{Méthodologie}

Une enquête téléphonique a été effectuée auprès d'une population de 2127 entreprises manufacturières et de services connexes provenant d'un répertoire de fabricants et de services du ministère du Développement économique, du Tourisme et de la Culture du Nouveau-Brunswick. Ā l'exception des filiales appartenant à des sociétés mères, toutes les entreprises du répertoire, quelle que soit leur taille, ont été contactées. À noter cependant que seulement 5 des 251 entreprises qui ont répondu à l'étude comptaient plus de 100 employés. Le critère des trois appels avant l'abandon a été retenu, c'est-à-dire que si le propriétaire ne pouvait pas être rejoint après trois tentatives, il était rayé de la liste. Si le propriétaire était rejoint et acceptait de participer à la recherche, il se voyait offrir le choix d'effectuer l'enquête par téléphone ou par la poste. S'il optait pour la poste, un questionnaire dans la langue de son choix, accompagné d'une enveloppe-réponse affranchie, lui était aussitôt envoyé. Un total de 251 questionnaires furent ainsi collectés pour un taux de réponse de $24,3 \%(251 \div 1032)$. Une fois les données des questionnaires compilées, des analyses statistiques ont été effectuées. Les résultats de ces analyses sont présentés dans la prochaine section.

Le questionnaire utilisé dans l'étude correspond à celui développé et validé par Djerdjour dans le cadre d'une étude sur les entreprises manufacturières ${ }^{6}$. Quelques modifications lui furent apportées pour l'adapter au secteur des services. Le questionnaire met 
en évidence les facteurs de la gestion de la qualité les plus souvent mentionnés dans la littérature sur l'adoption et la gestion des programmes de qualité.

\section{Résultats}

\section{Profil des entreprises}

Comme première étape, nous avons procédé à une analyse descriptive des données pour dégager les principales caractéristiques des entreprises de l'échantillon. Le tableau 1 présente ce profil selon deux groupes : les entreprises qui n'ont pas de système de qualité et celles qui en ont un. Le premier constat est que 213 entreprises sur un total de 251 (84,9\%) n'ont pas de programme formel de qualité, tandis que 38 entreprises $(15,1 \%)$ en ont implanté un. Un survol rapide des entreprises sans programme de qualité révèle que $57 \%$ sont anglophones et $43 \%$ sont francophones, $62 \%$ proviennent du sud de la province, $65 \%$ œuvrent principalement dans le domaine manufacturier, $87 \%$ ont moins de 16 employés, $78 \%$ ont un chiffre d'affaires inférieur à 1 million et $66 \%$ sont âgées de plus de 10 ans.

Tableau 1 - Profil des PME manufacturières et de services connexes du Nouveau-Brunswick

\begin{tabular}{|c|c|c|c|c|c|}
\hline \multicolumn{2}{|c|}{ Caractéristiques } & \multicolumn{2}{|c|}{$\begin{array}{l}\text { Entreprises sans un } \\
\text { programme de qualité }\end{array}$} & \multicolumn{2}{|c|}{$\begin{array}{c}\text { Entreprises avec un } \\
\text { programme de qualité }\end{array}$} \\
\hline & & $n=213$ & $\%$ & $\mathbf{N}=\mathbf{3 8}$ & $\%$ \\
\hline \multirow{2}{*}{ Langue } & Français & 91 & $43 \%$ & 22 & $58 \%$ \\
\hline & Anglais & 122 & 57 & 16 & 42 \\
\hline \multirow{3}{*}{ Région } & Région du sud & 120 & 62 & 20 & 57 \\
\hline & Région nord-est & 40 & 21 & 8 & 23 \\
\hline & Région nord-ouest & 34 & 17 & 7 & 20 \\
\hline \multirow{2}{*}{ Industrie } & Manufacturier & 138 & 65 & 29 & 76 \\
\hline & Service à l'entreprise & 73 & 35 & 9 & 24 \\
\hline \multirow{3}{*}{$\begin{array}{l}\text { Nombre } \\
\text { d'employés }\end{array}$} & 1 à 5 employés & 125 & 59 & 2 & 5 \\
\hline & 6 à 15 employés & 59 & 28 & 10 & 26 \\
\hline & 16 et plus & 28 & 13 & 26 & 69 \\
\hline \multirow{4}{*}{$\begin{array}{c}\text { Chiffre } \\
\text { d'affaires }\end{array}$} & Moins de 100000 & 48 & 26 & 1 & 3 \\
\hline & 100000 à 500000 & 70 & 38 & 3 & 8 \\
\hline & 500000 à 1 million & 25 & 14 & 8 & 21 \\
\hline & 1 million et plus & 40 & 22 & 26 & 68 \\
\hline \multirow{3}{*}{$\begin{array}{l}\text { Âge - } \\
\text { entreprise }\end{array}$} & 1 à 5 ans & 31 & 15 & 7 & 18 \\
\hline & 6 à 10 ans & 41 & 19 & 3 & 8 \\
\hline & 11 et plus & 139 & 66 & 28 & 74 \\
\hline \multirow{2}{*}{ Scolarité } & Secondaire & 67 & 32 & 10 & 26 \\
\hline & Post-secondaire & 141 & 68 & 28 & 74 \\
\hline \multirow{5}{*}{$\begin{array}{l}\text { Système de } \\
\text { qualité en } \\
\text { place }\end{array}$} & GIQ & - & - & 1 & 3 \\
\hline & ISO 9001 & - & - & 5 & 13 \\
\hline & ISO 9002 & - & - & 13 & 34 \\
\hline & Cercle de qualité & - & - & 5 & 13 \\
\hline & Inspection formelle & - & - & 14 & 37 \\
\hline
\end{tabular}

La même analyse effectuée chez les entreprises possédant un programme de qualité indique que la majorité des 38 entreprises qui ont participé à l'enquête sont francophones (58\%), proviennent également du sud de la province (57\%), œuvrent dans le domaine manufacturier $(76 \%)$, ont plusieurs employés (69\% des entreprises ont 16 employés et plus), ont un chiffre d'affaires supérieur à 1 million dans $68 \%$ des cas 
et sont en majorité âgées de plus de 10 ans (74\%). Les programmes de qualité les plus utilisés sont, dans l'ordre, les normes ISO 9000 (47\%), les systèmes d'inspection (37\%), les cercles de qualité (13\%) et la gestion intégrale de la qualité (3\%).

Ces derniers résultats démontrent bien la popularité des programmes d'assurance qualité ISO 9000 qui représentent $47 \%$ des systèmes de qualité en place. En revanche, on remarque que les programmes de qualité plus sophistiqués comme la gestion intégrale de la qualité et les cercles de qualité sont moins présents dans les entreprises du Nouveau-Brunswick. Un tel constat était attendu, considérant que la province du Nouveau-Brunswick, à l'image de notre échantillon, ne compte pas beaucoup d'entreprises de grande taille et qu'on retrouve généralement des systèmes de qualité plus sophistiqués dans les plus grandes entreprises.

Tableau 2 - Tests de moyennes

\begin{tabular}{|ccc|} 
& $\begin{array}{c}\text { Entreprise sans un } \\
\text { système de qualité }\end{array}$ & $\begin{array}{c}\text { Entreprise avec } \\
\text { système de qualité }\end{array}$ \\
\hline $\begin{array}{l}\text { Nombre d'employés* } \\
\text { Âge de l'entreprise** }\end{array}$ & 63,86 employés & 11,82 employés \\
$*: \mathrm{p}<0,01 ; * *: \mathrm{p}<0,05$. & 24,63 ans & 18,46 ans \\
\hline
\end{tabular}

Tableau 3 - Tests de Kolmokorov-Smirnov

\begin{tabular}{|c|c|c|c|c|}
\hline & \multicolumn{2}{|c|}{$\begin{array}{l}\text { Entreprise sans un } \\
\text { système de qualité }\end{array}$} & \multicolumn{2}{|c|}{$\begin{array}{l}\text { Entreprise avec } \\
\text { système de qualité }\end{array}$} \\
\hline & $\mathbf{n}=\mathbf{2 1 3}$ & $\%$ & $n=38$ & $\%$ \\
\hline \multicolumn{5}{|l|}{ Niveau de scolarité } \\
\hline Secondaire & 67 & 32 & 10 & 26 \\
\hline Post-secondaire & 141 & 68 & 28 & 74 \\
\hline \multicolumn{5}{|l|}{ Chiffre d'affaires* } \\
\hline Moins de 100000 & 48 & 26 & 1 & 3 \\
\hline 100000 à 250000 & 43 & 23 & 2 & 5 \\
\hline 250000 à 500000 & 27 & 15 & 1 & 3 \\
\hline 500000 à 1 million & 25 & 14 & 8 & 21 \\
\hline 1 million à 5 millions & 30 & 16 & 12 & 31 \\
\hline 5 millions à 10 millions & 5 & 3 & 5 & 13 \\
\hline Plus de 10 millions & 5 & 3 & 9 & 24 \\
\hline
\end{tabular}

Certains autres résultats obtenus de l'analyse des profils ne surprennent pas. Par exemple, le nombre élevé d'entreprises provenant du sud de la province s'explique par le fait qu'on retrouve une plus grande proportion d'entreprises dans cette région. Le fait également que le chiffre d'affaires et le nombre d'employés des entreprises sans programme de qualité soient plus modestes que ceux des entreprises avec un programme de qualité coïncide avec la tendance générale voulant qu'une entreprise doive généralement avoir une certaine taille pour justifier l'existence d'un système de qualité. À cet effet, des tests statistiques réalisés sur les variables chiffre d'affaires, nombre d'employés et âge de l'entreprise ont révélé une différence significative à un seuil de 0,05 (tableaux 2 et 3). Ainsi, l'âge de l'entreprise (nombre 
d'années d'existence) et la taille des entreprises de notre échantillon, mesurée par le nombre d'employés et le chiffre d'affaires, ont une incidence sur la présence d'un système de qualité dans l'entreprise. Cependant, une analyse effectuée sur la variable scolarité n'a pas révélé de différence statistiquement significative (tableau 3). C'est donc dire que le niveau de scolarité n'a pas été, dans notre étude, une variable permettant d'expliquer la présence ou l'absence de programme de qualité au sein de l'entreprise.

Pour terminer, un test d'indépendance du khi carré a été effectué sur les variables langue (français, anglais), région (sud, nord-est, nord-ouest) et industrie (manufacturier, service) des entreprises possédant un programme de qualité par rapport à celles qui n'en ont pas pour tester l'existence d'une relation. Les résultats de cette analyse ne révèlent aucune différence statistiquement significative $(p>0,05$, méthode d'estimation statistique de Pearson) entre les trois va- riables dépendantes (langue, région et industrie) et la variable dépendante (présence ou absence d'un système formel de qualité).

\section{Attitude des dirigeants envers les systèmes de qualité}

Le tableau 4 présente certaines caractéristiques liées à l'attitude des dirigeants d'entreprises qui n'ont pas de système de qualité. Un premier constat qui ressort de ce tableau est que la qualité est un élément très important pour les entreprises n'ayant pas implanté de système de qualité. En effet, $94 \%$ des répondants ont dit que la qualité était soit importante, soit cruciale pour leur entreprise. Deuxièmement, les raisons les plus souvent citées pour expliquer l'absence de système de qualité sont, dans l'ordre : la petite taille de l'entreprise $(22 \%)$, la qualité n'est pas exigée dans leur industrie $(21 \%)$, les coûts élevés $(16 \%)$ et le fait que la qualité ne constitue pas un problème $(13 \%)$.

Tableau 4 - Attitude des dirigeants d'entreprise sans système de qualité

\begin{tabular}{|c|c|c|c|}
\hline Système & Attitude & $\mathbf{n}=213$ & $\%$ \\
\hline \multirow{4}{*}{$\begin{array}{l}\text { Importance de la } \\
\text { qualité }\end{array}$} & Pas importante & 4 & 2 \\
\hline & Quelque peu importante & 9 & 4 \\
\hline & Importante & 45 & 21 \\
\hline & Cruciale & 155 & 73 \\
\hline \multirow{9}{*}{$\begin{array}{l}\text { Raisons pour ne pas } \\
\text { avoir implanté un } \\
\text { système de qualité }\end{array}$} & L'entreprise est trop petite & 40 & 22 \\
\hline & La qualité n'est pas exigée par l'industrie & 37 & 21 \\
\hline & Les coûts sont trop élevés & 28 & 16 \\
\hline & La qualité n'est pas un problème & 23 & 13 \\
\hline & $\begin{array}{l}\text { Le dirigeant n'a pas assez d'informations et } \\
\text { de connaissances }\end{array}$ & 16 & 9 \\
\hline & Il y a un manque de temps & 14 & 8 \\
\hline & L'entreprise a son propre système informel & 15 & 8 \\
\hline & Le dirigeant est nouveau en affaires & 3 & 2 \\
\hline & Les employés résistent à l'implantation & 1 & 1 \\
\hline \multirow{3}{*}{$\begin{array}{l}\text { Intention d'implanter } \\
\text { un système de qualité } \\
\text { dans l'avenir }\end{array}$} & Oui & 57 & 28 \\
\hline & Non & 117 & 58 \\
\hline & Peut-être & 28 & 14 \\
\hline \multirow{4}{*}{$\begin{array}{l}\text { Système de qualité } \\
\text { préféré }\end{array}$} & Gestion intégrale de la qualité & 17 & 19 \\
\hline & ISO 9000 & 61 & 67 \\
\hline & Inspection formelle & 8 & 9 \\
\hline & Cercle de qualité & 5 & 5 \\
\hline
\end{tabular}


Parmi les raisons les moins populaires, mentionnons la résistance des employés (1\%) et le fait que le dirigeant vient de débuter en affaires $(2 \%)$. Les données collectées sur les raisons pour expliquer l'absence d'un système de qualité corroborent celles obtenues par d'autres études, à savoir le coût élevé d'implantation et la petite taille de l'entreprise ${ }^{7}$.

Par ailleurs, l'implantation d'un système de qualité est envisagée par $42 \%$ des dirigeants d'entreprise. Lorsque questionnés sur le système de qualité qu'ils privilégiaient, $67 \%$ de ces mêmes répondants ont dit favoriser les normes ISO 9000, $19 \%$ des répondants préféraient la gestion intégrale de la qualité, tandis que les deux derniers groupes de répondants optaient pour l'inspection formelle $(9 \%)$ et les cercles de qualité (5\%).

Ces résultats démontrent bien que la qualité est un élément important, même pour les entreprises n'ayant pas de système de qualité, et que le programme d'assurance qualité ISO 9000 demeure le programme le plus populaire auprès des entreprises de notre échan- tillon. Ce dernier résultat peut s'expliquer par le fait que l'implantation des normes ISO est essentiellement l'étape la plus facile à franchir pour une entreprise qui désire passer d'un système d'inspection (ou d'aucun système de qualité) à un système de qualité beaucoup plus structuré.

Le tableau 5 présente certains éléments de l'attitude des dirigeants d'entreprises qui ont un système de qualité en place. À cet effet, les 38 entreprises de l'échantillon équipées d'un système de qualité identifient la qualité comme étant importante ou cruciale au sein de leur organisation. De plus, aucune entreprise n'a l'intention d'abandonner son système de qualité actuel. Au contraire, la majorité d'entre elles (56\%) projettent de l'améliorer. À ce sujet, le programme ISO 9000 est encore une fois le programme le plus souvent mentionné par les répondants (42\%). Quant aux résultats obtenus sur les coûts de l'implantation d'un système de qualité, ils sont partagés et semblent indiquer que les coûts d'implantation fluctuent selon le type de système de qualité retenu.

Tableau 5 - Attitude des dirigeants avec un système de qualité

\begin{tabular}{|c|c|c|c|}
\hline Système & Attitude & $\mathbf{n}=38$ & $\%$ \\
\hline \multirow{4}{*}{$\begin{array}{l}\text { Importance de } \\
\text { la qualité }\end{array}$} & Pas importante & - & - \\
\hline & Quelque peu importante & - & - \\
\hline & Importante & 16 & 42 \\
\hline & Cruciale & 22 & 58 \\
\hline \multirow{4}{*}{$\begin{array}{l}\text { Plans futurs pour } \\
\text { le(s) systèmes de } \\
\text { qualité déjà en } \\
\text { place }\end{array}$} & Maintenir le statu quo & 14 & 39 \\
\hline & Abandonner le système de qualité actuel & - & - \\
\hline & Améliorer le système de qualité actuel & 20 & 56 \\
\hline & Introduire un nouveau système de qualité & 2 & 5 \\
\hline \multirow{4}{*}{$\begin{array}{l}\text { Systèmes de } \\
\text { qualité favorisés } \\
\text { pour l'avenir }\end{array}$} & Gestion intégrale de la qualité & 1 & 2 \\
\hline & ISO 9000 & 18 & 42 \\
\hline & Cercle de qualité & 5 & 12 \\
\hline & Inspection formelle & 14 & 33 \\
\hline \multirow{5}{*}{$\begin{array}{c}\text { Coûts de } \\
\text { l'implantation } \\
\text { du système de } \\
\text { qualité }\end{array}$} & Aucun & 3 & 9 \\
\hline & Moins de 10000 & 9 & 26 \\
\hline & Entre 10000 et 50000 & 9 & 26 \\
\hline & Entre 50000 et 100000 & 10 & 28 \\
\hline & Plus de 100000 & 4 & 11 \\
\hline
\end{tabular}

\section{Raisons, impacts et difficultés associés à l'implantation d'un système de qualité}

Dans un premier temps, on observe au tableau 6a que les facteurs qui ont le plus contribué à la décision d'implanter un système de qualité sont la volonté de mieux satisfaire la clientèle $(4,57)$, le désir d'améliorer la qualité des produits $(4,56)$ ainsi que celui d'améliorer l'image de l'entreprise $(3,97)$, tandis que la 
pression des concurrents $(2,50)$ ainsi que le fait de rattraper les concurrents $(2,22)$ ont été les facteurs les moins importants. Les données collectées sur les facteurs qui ont le plus contribué à la décision d'implan- ter un système de qualité appuient les raisons relevées dans la littérature, à savoir la satisfaction de la clientèle et le désir d'améliorer l'image de l'entreprise ${ }^{8}$.

Tableau 6a - Raisons invoquées pour l'implantation d'un système de qualité

\begin{tabular}{l|lr}
\hline & Satisfaire le client & 4,57 \\
& Améliorer la qualité des produits et des services & 4,56 \\
& Améliorer l'image de l'entreprise & 3,97 \\
& Réaliser une plus grande accessibilité au marché & 3,91 \\
Raisons pour lesquelles un & Diminuer le gaspillage et les coûts de réusinage & 3,61 \\
système de qualité a été & Répondre aux pressions des clients & 3,17 \\
implanté & Capturer de meilleures affaires dans les marchés & 2,93 \\
& étrangers & 2,55 \\
& Introduire un nouveau système de gestion & 2,50 \\
& Rattraper les concurrents & 2,22
\end{tabular}

Par ailleurs, les impacts positifs les plus ressentis par les entreprises ayant implanté un système de qualité (tableau 6b) sont une production plus efficiente $(3,94)$, une réduction du nombre de plaintes de la clientèle $(3,94)$, une meilleure gestion $(3,89)$ et une production plus élevée $(3,82)$. À noter que l'amélioration des profits arrive en dernier lieu avec un score de 3,29 Quant aux problèmes les plus souvent associés à l'implantation d'un système de qualité (tableau $6 c)$, on retrouve la résistance des employés $(3,14)$, les coûts trop élevés $(3,07)$, une durée d'implantation trop longue $(2,77)$ et un manque d'expertise dans le domaine de la qualité $(2,64)$. Le principal problème identifié par les répondants, soit la résistance des employés, ne surprend pas dans la mesure où il est souvent cité dans la littérature comme un des obstacles à l'implantation d'un système de qualité ${ }^{9}$. Plus particulièrement, ce problème met en perspective l'importance qu'on doit apporter à la formation des employés et au support offert après la formation de façon à augmenter les chances de succès de l'implantation d'un système de qualité.

Finalement, on remarque au tableau $6 \mathrm{~d}$ que les dirigeants eux-mêmes constituent la meilleure source d'assistance lors de l'implantation d'un système de qualité. Cela signifie que les dirigeants ont bien pris soin de s'informer avant de se lancer dans l'implantation d'un programme de qualité et démontrent bien leur engagement à la réalisation d'un tel projet.

Tableau 6b - Impacts de l'implantation d'un système de qualité

\begin{tabular}{|c|c|c|}
\hline \multirow{6}{*}{$\begin{array}{l}\text { Impacts du système de } \\
\text { qualité sur l'entreprise }\end{array}$} & Production plus efficiente & 3,94 \\
\hline & Moins de plaintes des clients & 3,94 \\
\hline & Meilleure gestion & 3,89 \\
\hline & Production plus élevée & 3,82 \\
\hline & Meilleur moral des employés & 3,58 \\
\hline & Profits améliorés & 3,29 \\
\hline
\end{tabular}


Tableau 6c - Difficultés rencontrées lors de l'implantation d'un système de qualité

\begin{tabular}{|c|c|c|}
\hline \multirow{6}{*}{$\begin{array}{l}\text { Problèmes rencontrés durant } \\
\text { l'implantation du système de } \\
\text { qualité }\end{array}$} & Résistance au changement - employés & 3,14 \\
\hline & Coûts élevés & 3,07 \\
\hline & Durée de l'implantation assez longue & 2,77 \\
\hline & Manque d'expertise dans le domaine & 2,64 \\
\hline & Trop de changements pour l'entreprise & 2,36 \\
\hline & Manque d'engagement du management & 1,93 \\
\hline
\end{tabular}

Tableau 6d - Sources d'assistance durant l'implantation d'un système de qualité

$\begin{array}{lll} & \text { Soi-même } & 4,88 \\ & \text { Consultants locaux (région immédiate) } & 3,05 \\ \text { Sources d'assistance durant } & \text { Gouvernement } & 2,93 \\ \text { l'implantation du système de } & \text { Consultants d'une autre province } & 2,18 \\ \text { qualité } & \text { Autres consultants de la province } & 1,90 \\ & \text { Consultants d'autres pays } & 1,50\end{array}$

\section{Conclusion}

Nous avons tenté, dans cet article, de dresser le profil des PME manufacturières et des services connexes du Nouveau-Brunswick ainsi que des systèmes de qualité mis en place dans ces entreprises. De plus, nous avons examiné l'attitude des propriétaires-dirigeants envers les systèmes formels de qualité ainsi que les difficultés rencontrées lors de leur implantation. Les résultats obtenus à partir de nos analyses démontrent que, parmi les variables démographiques retenues pour tracer le profil des entreprises, seules les variables taille de l'entreprise (mesurée par le nombre d'employés et le chiffre d'affaires) et l'âge de l'entreprise (nombre d'années d'existence) diffèrent entre les entreprises possédant un système et celles n'en possédant pas.

Ainsi, nos résultats révèlent que plus les entreprises sont âgées et plus elles ont un chiffre d'affaires et des effectifs élevés, plus elles sont susceptibles de posséder un système formel de qualité. À l'opposé, aucune relation statistiquement significative n'a été observée chez les autres variables démographiques de l'étude (langue, région et industrie).
Par ailleurs, les résultats obtenus indiquent que les systèmes de qualité ne sont pas très répandus dans les PME manufacturières et de services connexes de la province du Nouveau-Brunswick: seulement $15 \%$ des entreprises ayant participé à l'étude ont un système de qualité. C'est donc dire que $85 \%$ des entreprises interviewées n'ont aucun programme formel de qualité en place, malgré qu'elles perçoivent toutes la qualité comme un facteur important. Ce dernier résultat indique que ces répondants ne se sentent pas obligés de mettre en place un système de qualité. Les raisons pour expliquer ce comportement peuvent être liées aux perceptions de croissance de l'entreprise, à la vision et aux ambitions des dirigeants, au secteur dans lequel est situé l'entreprise, aux exigences des fournisseurs, etc. C'est donc avant tout une question de pertinence, d'obligation, de coûts/bénéfices et de justification.

Par contre, un point encourageant ressort de l'étude : la très grande majorité des entreprises, y compris celles n'ayant pas de systèmes de qualité, considèrent le concept de qualité comme une fonction très importante ou cruciale au sein de l'entreprise. D'ailleurs, plusieurs des entreprises sans système de qualité 
(42\%) envisageaient d'en implanter un dans le futur. Sur ce point, le système de qualité le plus souvent cité est la norme ISO 9000.

En guise de conclusion, notre étude révèle que de plus en plus de PME du Nouveau-Brunswick utilisent ou prévoient utiliser des systèmes de gestion de la qualité dans leur entreprise. L'implantation d'un système de qualité peut constituer une activité stratégique intéressante pour les PME du Nouveau-Brunswick ou pour toutes les PME qui désirent améliorer leur performance organisationnelle. À cet effet, la souplesse des structures des PME, la rapidité dans la prise de décision, le potentiel d'innovation dont elles disposent constituent des atouts et des conditions favorables à l'implantation d'une démarche de gestion de la qualité.

\section{Notes et références}

1 Cette recherche fut rendue possible grâce à une subvention de l'Association des universités partiellement ou entièrement de langue française - Universités des réseaux d'expression française (AUPELF-UREF). Les auteurs tiennent également à remercier Stéphanie Meunier pour sa précieuse collaboration à la réalisation de cette étude.

2 Yves Robichaud est professeur à l'École de l'administration et du commerce de l'Université Laurentienne à Sudbury, Ontario; Egbert McGraw est professeur à la faculté d'administration de l'Université de Moncton, au NouveauBrunswick; Mohamed Djerdjour est professeur à la State University of New York.

3 Ansari, M. (1999). «Les mesures de perception de la qualité totale dans les PMI: résultats de recherche », Revue internationale PME, vol. 12, $\mathrm{n}^{\circ}$ 4, p. 18-31; Tan, W. et S. Tay (1994). «Factors Contributing to the Growth of SMEs : The Singapore Case ", Proceedings of the Fifth ENDEC World Conference on entrepreneurship, Singapore, NTU-Entrepreneurship Development Center, p. 150161; Roth, R. et J. Miller, (1992). "Success Factors in Manufacturing », Business Horizons, vol. 35, p. 73-81.

4 Kuratko, D.F., J.C. Goodale et J.S. Hornsby (2001). "Quality Practices for a Competitive Advantage in Smal- ler Firms ", Journal of Small Business Management, vol. 39, n 4, p. 293-311; Ansari, M. (1999), op. cit.; Messenghem, K. (1999). «L'assurance qualité : facteur dénaturant de la PME ", Revue internationale PME, vol. 12, $\mathrm{n}^{\circ} 3$, $\mathrm{p}$. 107-126; Hodgetts, R.M., D.F. Kuratko et J.S. Hornsby, J.S. (1999). «Quality Implementation in Small Business : Perspectives from the Baldridge Award Winners », SAM Advanced Management Journal, vol. 64, $\mathrm{n}^{\circ}$ 1, p. 37-47; Messeghem, K. et N. Varraut (1998). « Stratégies d'adoption d'une démarche qualité en PME », Revue internationale PME, vol. 11, $\mathrm{n}^{\circ}$ 1, p. 101-122; Shea, J. et D. Gobeli (1995). «TQM : The Experiences of Ten Small Businesses », Business Horizons, vol. 38, n 1, p. 83-97.

5 Thurow, L.C. (1980). The zero-sum society, New York, Penguin Books; Cole, R.E. (1999). Managing Quality Fads: How American Business Learned How to Play the Quality Game, New York, Oxford University Press.

6 Djerdjour, M. (2000). « An Exploratory Study of Quality in the Manufacturing Sector of a Small Developing Country ", Proceedings of ASAC-IFSAM conference, Production and Operations Management, vol. 21, $\mathrm{n}^{\circ}$, p. 12-23.

7 North, J., J. Curran et R. Blackburn (1993). « Small Firms and ISO 9000 : A Preliminary Investigation $" 16^{\text {th }}$ National Small Firms Policy and Research Conference, Nottingham.

8 Chittenden, F., P. Poutziouris et S.M. Mukhtar (1998). «Small Firms and the ISO 9000 Approach to Quality Management », International Small Business Journal, vol. 17, n 1, p. 73-88; North, J., J. Curran et R. Blackburn, R. (1993), op.cit.; Kakkar, S.K. (1995). «ISO 9000 and TQM : An Indian Scenario », Pakistan First International Convention on Quality Control, Pakistan Institute of Quality Control.

9 Laflamme, R. et M. Pelletier (1995). «La gestion intégrale de la qualité : critères de succès ", Revue Organisation, vol. 4, $\mathrm{n}^{\circ}$ 2, p. 23-44; Djerdjour, M. et R. Patel (2000). " Implementation of Quality Programmes in Developing Countries : a Fiji Islands Case Study », Total Quality Management, vol. 11, $\mathrm{n}^{\circ}$ 1, p. 25-44; Harrington, D. et G. Akehurst (2000). «An Empirical Study of Service Quality Implementation », The Service Industries Journal, vol. 20, $\mathrm{n}^{\circ}$ 2, p. 133-156; Kakkar, S.K. (1995), op. cit. 


\section{PUBLICITÉ}

Quelle est votre opinion ? 